\title{
A new pathological assessment method to assess residual lesions after neoadjuvant chemotherapy for breast cancer, residual disease in breast and nodes combined with Ki-67 (RDBN-K)
}

\author{
Ruoqi Han ${ }^{1}$, Yanqi Ma ${ }^{2}$, Zhikun Liu ${ }^{3}$, Chunxiao $\mathrm{Li}^{1}$, Sainan $\mathrm{Li}^{1}$, Xiangshun $\mathrm{Kong}^{4}$, Meng $\mathrm{Han}^{5}$, \\ Yueping Liu ${ }^{2}$, Cuizhi Geng ${ }^{1}$
}

${ }^{1}$ Department of Breast Center, The Fourth Hospital of Hebei Medical University, Shijiazhuang, China; ${ }^{2}$ Department of Pathology, The Fourth Hospital of Hebei Medical University, Shijiazhuang, China; ${ }^{3}$ Department of Radiotherapy, The Fourth Hospital of Hebei Medical University, Shijiazhuang, China; ${ }^{4}$ Department of Breast Surgery, Xingtai People's Hospital, Xingtai, China; ${ }^{5}$ Department of Breast Surgery, The First Hospital of Qinhuangdao, Qinhuangdao, China

Contributions: (I) Conception and design: R Han, Y Ma, Y Liu, C Geng; (II) Administrative support: Y Liu, C Geng; (III) Provision of study materials or patients: All authors; (IV) Collection and assembly of data: All authors; (V) Data analysis and interpretation: R Han, Z Liu; (VI) Manuscript writing: All authors; (VII) Final approval of manuscript: All authors.

Correspondence to: Dr. Cuizhi Geng. Department of Breast Center, The Fourth Hospital of Hebei Medical University, 12 Health Road, Shijiazhuang 050011, China. Email: cuizhigeng@hotmail.com; Dr. Yueping Liu. Department of Pathology, The Fourth Hospital of Hebei Medical University, 12 Health Road, Shijiazhuang 050011, China. Email: annama@163.com.

Background: The accurate assessment of residual tumor tissue after neoadjuvant chemotherapy (NAC) for breast cancer is closely related to the subsequent treatment and prognosis of patients. The objective of this study is to develop a new pathological assessment metric for breast cancer patients through combining residual disease in breast and nodes (RDBN) and the Ki-67 expression status after NAC. We call the new metric residual disease in breast and nodes combined with Ki-67 (RDBN-K) and aim to study its significance for prognosis.

Methods: A total of 723 breast cancer patients with TNM staging II to III who received NAC and surgical treatment underwent residual disease evaluation by RDBN-K and RDBN. All patients were followed up for a median of 44 months. We used pairwise stratified analysis to compare the accuracy and clinical significance of the RDBN and RDBN-K.

Results: Pairwise stratified analysis revealed that DFS and OS had larger difference between RDBN-K-3 and RDBN-K-4 compared to between RDBN-3 and RDBN-4. Moreover, RDBN-K also showed larger differences in OS between stage 2 and 3 compared to RDBN alone.

Conclusions: Incorporating Ki-67 expression status into RDBN improved the accuracy in residual tumor burden assessment after NAC. RDBN-K is a better metric for predicting treatment outcomes and identify patients who warrant follow-up intensive treatment.

Keywords: Residual disease in breast and nodes (RDBN); residual disease in breast and nodes combined with Ki67 (RDBN-K); neoadjuvant chemotherapy (NAC); Ki-67; prognosis

Received: 14 December 2021; Accepted: 19 January 2022; Published: 30 July 2022.

doi: $10.21037 /$ tbcr-21-39

View this article at: https://dx.doi.org/10.21037/tbcr-21-39 


\section{Introduction}

The accurate assessment of residual tumor after neoadjuvant chemotherapy (NAC) is essential to inform subsequent treatment plans and disease prognosis for breast cancer patients. The pathological response of the primary tumor to chemotherapy has previously been proven to be a significant prognostic marker (1). The complete pathological response (pCR) after NAC has been bound up with 5-year survival rates of up to $87 \%$ (2). When no residual invasive cancer in the breast exists after NAC, the number of metastatic lymph nodes is negatively correlated with survival (3). Patients with a lymph node-negative status after chemotherapy have excellent survival even with residual breast disease (4). A recent study by Choi et al. (5) showed that a higher histological grade after NAC was associated with a worse prognosis. Common methods assessing residual diseases after NAC include residual cancer burden (RCB), MillerPayne (MP) classification, and the residual disease in breast and nodes (RDBN), each with distinct focuses and advantages. Among them, RDBN was first proposed in 2008 (6) and combined the residual breast tumor size, lymph node metastasis status and tumor histological grade after NAC to form a single prognostic parameter. Multiple groups have reported RDBN is closely related to the prognosis (5,7-11). Compared with other assessment methods, it shows improved the accuracy; but it is not ideal.

Ki-67, a cell proliferation marker, has been widely used in predicting invasive breast cancer prognosis and making treatment decisions. Recent studies have shown that $\mathrm{Ki}$ 67 expression after NAC is an independent prognostic factor for breast cancer patient survival (12) and a powerful prognostic indicator for patients who have not reached pCR $(13,14)$. Patients with a high Ki-67 value in residual tumors after chemotherapy are closely correlated with a poor prognosis $(12,15-17)$.

Therefore, this study aims to examine the roles of residual breast tumor size, lymph node metastasis status and tumor histological grade after NAC, as well as Ki-67 expression status in reflecting tumor proliferation activity. These parameters are combined to form a new pathological assessment metric named residual disease in breast and nodes combined with Ki-67 (RDBN-K). We expect that RDBN-K can more accurately predict the prognosis of breast cancer patients after NAC. We present the following article in accordance with the REMARK reporting checklist (available at https://tbcr.amegroups.com/article/ view/10.21037/tbcr-21-39/rc).

\section{Methods}

\section{Patients}

The study retrospectively analyzed breast cancer patients who were treated in the galactophore department of three large comprehensive hospitals in Hebei Province from February 2013 to September 2017. The treatment plan of each patient was carried out in accordance with the Chinese guidelines for the diagnosis and treatment of breast cancer. Our inclusion criteria were as follows: (I) pathologically diagnosed female patients with invasive breast cancer; (II) primary breast cancer at age $\geq 18$ years as the initial diagnosis; (III) clinical TNM staging before NAC of stage II-III; (IV) successful completion of NAC of the expected course of treatment; (V) surgery. The exclusion criteria were as follows: (I) other malignant tumors; (II) distant metastasis during neoadjuvant therapy; (III) breast tumor resection before NAC; (IV) no axillary lymph node dissection; (V) incomplete postoperative pathological data and follow-up results. Finally, 723 patients were included in this study.

\section{Clinical data}

Clinical data were retrospectively reviewed from the electronic medical records. Primary tumors in the breast and the lymph node status were assessed by physical examination, bilateral mammogram, ultrasound, and magnetic resonance imaging (MRI) at the initial diagnosis. The diagnosis of primary breast cancer was established by core-needle biopsy, and lymph node metastasis was confirmed by core-needle biopsy or fine-needle aspiration. The patient's systemic condition was assessed by chest, abdominal and pelvic computed tomography (CT) and bone scan before NAC and before surgery, and positron emission tomography (PET) examination was performed if necessary. Pregnancy testing and counseling for fertility concerns were also necessary for all women of childbearing potential. During NAC, the residual tumor was assessed by physical examination, bilateral mammogram and breast MRI. Additionally, comprehensive metabolic tests were required, including liver function tests and alkaline phosphatase tests. Finally, the appropriate surgical treatment was selected after completion of the expected chemotherapy. Local and distant recurrence and metastasis were confirmed by histology or 

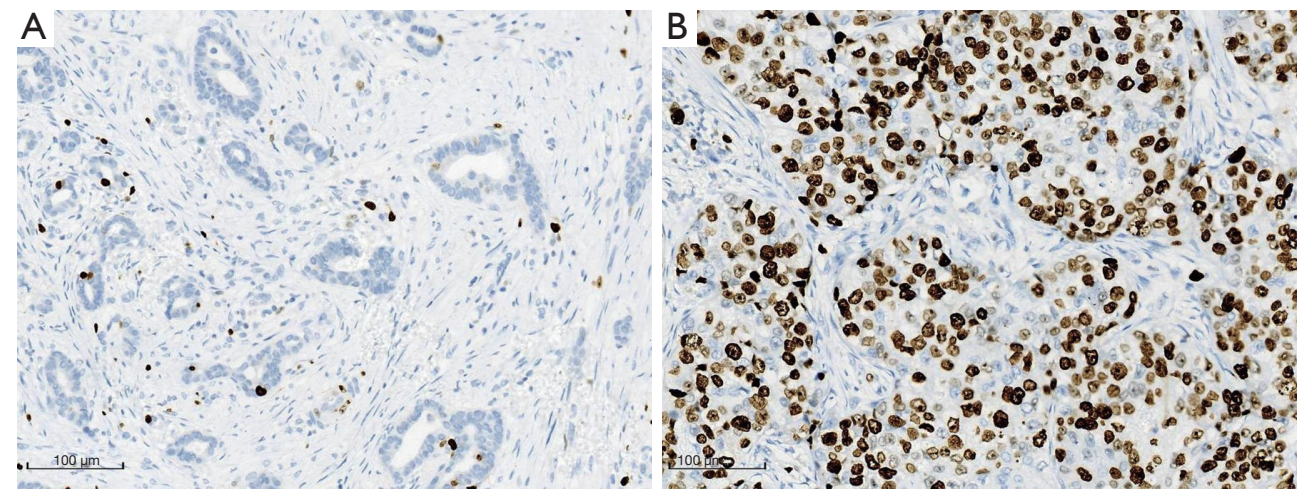

Figure 1 Ki-67 immunohistochemical staining (20×). (A) Ki-67 <14\%; (B) Ki-67 $\geq 14 \%$.

cytology. Bone metastasis was confirmed by imaging.

\section{Pathological data}

All tumor tissues were preserved in the form of wax blocks. The tumor specimens were independently evaluated by two experienced pathologists without knowing the endpoint of the study. RDBN is divided into four prognostic levels by applying the following formula, $\mathrm{RDBN}=0.2$ (residual breast tumor size in centimeters) + index of involved nodes + tumor histological grade (6). The residual tumor size is the largest diameter of tumor lesions in gross specimens, measured in centimeters $(\mathrm{cm})$. If the tumor bed was not obvious or difficult to determine, the position and size of the tumor bed were found using an X-ray camera. Finally, the microscopic results were combined with the gross view. The index of the involved lymph nodes is 0 for zero positive nodes, 1 for one to four positive nodes, 2 for between five and seven positive nodes, and 3 for eight or greater than eight positive lymph nodes. Isolated tumor cells (ITCs) in the lymph nodes after NAC were also considered lymph node positive. Tumor histological grading was according to the Nottingham grade, which stratifies tumors into grades I to III (18). The RDBN levels were defined as the following, a score of 0 corresponds to RDBN-1 (equivalent to pCR), RDBN-2 is between 0.1 and 2.9, RDBN-3 is between 3.0 and 4.3 , and RDBN-4 is 4.4 or above.

The residual disease in the breast and nodes combined with Ki-67 (RDBN-K) is calculated using the following formula: RDBN-K = RDBN + index of Ki-67. The Ki-67 index is scored according to the percentage of carcinoma cells with nuclear staining determined by accurate counting according to the International $\mathrm{Ki}-67$ in Breast Cancer Working Group recommendations (19). The index is 0 for less than $14 \%$ and 1 for $14 \%$ and above (Figure 1). If the distribution of stained cells was homogeneous, at least three infiltrating high magnification field counts are randomly selected to obtain an average Ki-67 index. If the distribution of stained cells was not homogeneous, distinct staining areas (hot spots) are observed, and at least 3 hot spots and 500 cells need to be evaluated. NAC can reduce tumor cells. Therefore, it could be difficult to detect 500 cells in certain hypo-cellular samples although increasing fields of view, and the standard were revised to accept a score of at least 200 cells. According to all calculated values of RDBN-K, two critical points were determined by the quartile method (16), and 3 (25th percentile point) was selected as the dividing point between RDBN-K-2 and RDBN-K-3, and 5.3 (75th percentile point) as the dividing point between RDBN-K-3 and RDBN-K-4. In summary, RDBN-K was divided into levels 1 to 4 . RDBN-K-1 (equivalent to pCR) corresponds to $\mathrm{RDBN}-\mathrm{K}=0, \mathrm{RDBN}-2$ is between 0.1 and $3, \mathrm{RDBN}-3$ is between 3.1 and 5.3, and RDBN-4 is 5.3 or above.

\section{Follow-up method}

A comprehensive follow-up method combining telephone follow-up, outpatient review and hospitalization was adopted. The follow-up interval was every 3 months for the first 2 years, every 6 months for the third to fifth years, and annually after 5 years. The end point of follow-up was December 31st, 2019. Overall survival (OS) was defined as the time from the date of first diagnosis to follow-up or the date of death from any reason. Disease-free survival (DFS) was defined as the time from the date of curative operative treatment to the date of recurrence, metastasis or follow-up. 
Table 1 Clinical Characteristics

\begin{tabular}{|c|c|}
\hline Characteristic & No. (\%) \\
\hline \multicolumn{2}{|l|}{ Age, years } \\
\hline$\leq 35$ & $78(10.8)$ \\
\hline $35-55$ & $431(59.6)$ \\
\hline$>55$ & $214(29.6)$ \\
\hline \multicolumn{2}{|l|}{ Protocols of NAC } \\
\hline AC-T & $89(12.3)$ \\
\hline TA & $305(42.2)$ \\
\hline TAC & $221(30.6)$ \\
\hline Including targeted therapy & $6(0.8)$ \\
\hline Others & $102(14.1)$ \\
\hline \multicolumn{2}{|l|}{ Pretherapy cT stage } \\
\hline T0 & $7(1.0)$ \\
\hline $\mathrm{T} 1$ & $54(7.5)$ \\
\hline T2 & $388(53.7)$ \\
\hline T3 & $128(17.7)$ \\
\hline $\mathrm{T} 4$ & $146(20.2)$ \\
\hline \multicolumn{2}{|l|}{ Pretherapy cN stage } \\
\hline No & $39(5.4)$ \\
\hline $\mathrm{N} 1$ & $257(35.5)$ \\
\hline N2 & $189(26.1)$ \\
\hline N3 & $238(32.9)$ \\
\hline \multicolumn{2}{|l|}{ Pretherapy clinical stage } \\
\hline$\| \mathrm{A}$ & $34(4.7)$ \\
\hline IIB & $152(21.0)$ \\
\hline IIIA & $214(29.6)$ \\
\hline IIIB & $85(11.8)$ \\
\hline IIIC & $238(32.9)$ \\
\hline
\end{tabular}

\section{Statistical analysis}

Statistical analyses were performed using SPSS statistical software. Descriptive analysis was used to analyze the clinical and pathological characteristics. Cox proportional hazards regression model was used to analyze the prognostic factors of pathological characteristics by univariate and multivariate analyses to evaluate the prognostic survival factors. The Kaplan-Meier survival curve (log-rank test) was used to analyze the prognosis of patients according to the pathological evaluation results. A $\mathrm{P}$ value less than 0.05 was considered as statistically significant.

\section{Ethical statement}

The study was conducted in accordance with the Declaration of Helsinki (as revised in 2013). This study was approved by Institutional Review Board of The Fourth Hospital of Hebei Medical University (No. 2020KY112). Informed consent was obtained from all individual participants included in the study.

\section{Results}

The clinical and pathological characteristics of enrolled patients are summarized in Tables 1,2. The median age of the patients was 50 years. Eighty-six patients (11.9\%) had a pCR in both primary breast lesions and regional lymph nodes. During the follow-up period, 147 (20.3\%) recurrences or metastases, $58(8.0 \%)$ local recurrences, $103(14.2 \%)$ distant metastasis and $69(9.5 \%)$ deaths were observed in the cohort.

Table 3 shows that the Ki-67 expression status after NAC divided into two groups according to the defining criteria of $<14 \%$ (low expression) and $\geq 14 \%$ (high expression). The 3 -year DFS rates were $85.9 \%$ and $73.3 \%$ respectively, and the 3 -year OS rates were $96.9 \%$ and $90.1 \%$ respectively. Univariate prognosis analysis showed that the expression of Ki-67 after NAC was significantly different from the DFS and OS of patients (Table 4; DFS, $\mathrm{P}<0.001$; OS, $\mathrm{P}<0.001$ ). Multivariate prognosis analysis showed that the Ki-67 expression status after NAC was significantly different from the DFS and OS of patients (DFS, $\mathrm{P}=0.002$; OS, $\mathrm{P}<0.001$ ).

The relative risk levels of local recurrence and mortality calculated by both RDBN and RDBN-K are shown in Table 5. In RDBN, 86 (12\%), 122 (17\%), 295 (41\%), and $220(30 \%)$ of the 723 patients were grouped into RDBN1 (pCR), RDBN-2, RDBN-3, and RDBN-4, respectively. Compared to patients in $\mathrm{RDBN}-1$, the relative risks of recurrence or metastasis were $2.2(\mathrm{P}=0.080)$ for $\mathrm{RDBN}-2$, $2.8(\mathrm{P}=0.011)$ for $\mathrm{RDBN}-3$, and $4.0(\mathrm{P}=0.001)$ for RDBN4. The relative risk values of death were $2.8(\mathrm{P}=0.186)$ for RDBN-2, $4.9(\mathrm{P}=0.030)$ for $\mathrm{RDBN}-3$, and $6.7(\mathrm{P}=0.009)$ for RDBN-4. In the RDBN-K, 86 (12\%), 97 (13\%), 358 $(50 \%)$, and $182(25 \%)$ of the 723 patients were grouped into RDBN-K-1 (pCR), RDBN-K-2, RDBN-K-3 and 
Table 2 Pathological characteristics

\begin{tabular}{|c|c|}
\hline Characteristic & No. (\%) \\
\hline \multicolumn{2}{|l|}{ Pathological type } \\
\hline Invasive ductal carcinoma & $439(60.7)$ \\
\hline Invasive lobular carcinoma & $9(1.2)$ \\
\hline Metaplastic carcinoma & $7(1.0)$ \\
\hline Ductal and papillary & $17(2.4)$ \\
\hline Mucinous adenocarcinoma & $16(2.2)$ \\
\hline Other or unknown & $235(32.5)$ \\
\hline \multicolumn{2}{|c|}{ Posttherapy Nottingham tumor grade } \\
\hline Complete response & $105(14.5)$ \\
\hline Grade I & $28(3.9)$ \\
\hline Grade II & $443(61.3)$ \\
\hline Grade III & $147(20.3)$ \\
\hline \multicolumn{2}{|l|}{ Posttherapy node involvement } \\
\hline 0 & $212(29.3)$ \\
\hline $1-4$ & $291(40.2)$ \\
\hline $5-7$ & $91(12.6)$ \\
\hline$\geq 8$ & $129(17.8)$ \\
\hline \multicolumn{2}{|c|}{ Posttherapy lymph-vascular space invasion } \\
\hline Yes & $167(23.1)$ \\
\hline No & $556(76.9)$ \\
\hline \multicolumn{2}{|l|}{ Pathologic response } \\
\hline $\mathrm{pCR}$ & $86(11.9)$ \\
\hline No pCR & $637(88.1)$ \\
\hline \multicolumn{2}{|l|}{ ER status } \\
\hline Positive & $492(68.0)$ \\
\hline Negative & 231 (32.0) \\
\hline \multicolumn{2}{|l|}{ PR status } \\
\hline Positive & $411(56.8)$ \\
\hline Negative & $312(43.2)$ \\
\hline \multicolumn{2}{|l|}{ HER2 status } \\
\hline Positive & $303(41.9)$ \\
\hline Negative & $420(58.1)$ \\
\hline \multicolumn{2}{|l|}{ Posttherapy Ki-67 } \\
\hline$<14 \%$ & $257(35.5)$ \\
\hline$\geq 14 \%$ & $466(64.5)$ \\
\hline
\end{tabular}

pCR, pathologic complete response; ER, estrogen receptor; PR, progesterone receptor; HER2, human epidermal growth factor receptor 2 .
Table 3 Analysis of prognosis

\begin{tabular}{|c|c|c|}
\hline Characteristic & 3-year DFS (\%) & 3-year OS (\%) \\
\hline \multicolumn{3}{|c|}{ Posttherapy node involvement } \\
\hline 0 & 88.0 & 98.0 \\
\hline $1-4$ & 80.1 & 92.3 \\
\hline $5-7$ & 76.6 & 93.8 \\
\hline$\geq 8$ & 69.1 & 89.9 \\
\hline \multicolumn{3}{|c|}{ Posttherapy Nottingham tumor grade } \\
\hline Complete response & 88.0 & 99.0 \\
\hline Grade I & 82.1 & 96.4 \\
\hline Grade II & 78.5 & 94.0 \\
\hline Grade III & 78.4 & 90.8 \\
\hline \multicolumn{3}{|l|}{ Ki-67 } \\
\hline$<14 \%$ & 85.9 & 96.9 \\
\hline$\geq 14 \%$ & 73.3 & 90.1 \\
\hline \multicolumn{3}{|l|}{ RDBN } \\
\hline RDBN-1 & 91.5 & 98.8 \\
\hline RDBN-2 & 83.4 & 96.6 \\
\hline RDBN-3 & 80.9 & 93.9 \\
\hline RDBN-4 & 72.5 & 90.0 \\
\hline \multicolumn{3}{|l|}{ RDBN-K } \\
\hline RDBN-K-1 & 91.5 & 98.8 \\
\hline RDBN-K-2 & 87.4 & 99.0 \\
\hline RDBN-K-3 & 80.0 & 94.1 \\
\hline RDBN-K-4 & 70.6 & 87.9 \\
\hline
\end{tabular}

DFS, disease-free survival; OS, overall survival; RDBN, residual disease in breast and nodes; RDBN-K, residual disease in breast and nodes combined with $\mathrm{Ki}-67$.

RDBN-K-4, respectively. Using RDBN-K-1 as the reference value, the relative risk values of recurrence or metastasis were $1.7(\mathrm{P}=0.239)$ for $\mathrm{RDBN}-\mathrm{K}-2,2.8(\mathrm{P}=0.009)$ for RDBN-K-3, and $4.3(\mathrm{P}<0.001)$ for RDBN-K-4, and the relative risk values of death were $1.3(\mathrm{P}=0.744)$ for RDBN-K-2, $4.8(\mathrm{P}=0.032)$ for RDBN-K-3, and 8.0 $(\mathrm{P}=0.004)$ for $\mathrm{RDBN}-4$.

The results of the reclassification of RDBN categories when using the new RDBN-K metric are shown in Table 6. Patients with pCR (RDBN-1 and RDBN-K-1) were classified using both RDBN and RDBN-K calculations. Among cases which were classified as RDBN-2, 40 (5.5\%) 
Table 4 Univariate and multivariate analyses of factors predictive for disease-free survival and overall survival

\begin{tabular}{|c|c|c|}
\hline Variables & Univariate analysis $P$ value & Multivariate analysis $P$ value \\
\hline Posttherapy node involvement & $<0.001$ & - \\
\hline Posttherapy Nottingham tumor grade & 0.085 & - \\
\hline Posttherapy Ki-67 & $<0.001$ & 0.002 \\
\hline \multicolumn{3}{|l|}{ os } \\
\hline Posttherapy node involvement & 0.006 & - \\
\hline Posttherapy Nottingham tumor grade & 0.035 & - \\
\hline Posttherapy Ki-67 & $<0.001$ & $<0.001$ \\
\hline
\end{tabular}

$\mathrm{P}<0.05$ is considered significant. DFS, disease-free survival; OS, overall survival.

Table 5 Relative risk of recurrence and mortality using RDBN and RDBN-K score categories

\begin{tabular}{|c|c|c|c|c|c|c|c|}
\hline $\begin{array}{l}\text { Score } \\
\text { category }\end{array}$ & No. & \multicolumn{3}{|c|}{ Overall recurrence } & \multicolumn{3}{|c|}{ Mortality } \\
\hline \multicolumn{8}{|l|}{ RDBN } \\
\hline 1 & 86 & $7(8.1)$ & 1.0 & & $2(2.3)$ & 1.0 & \\
\hline 2 & 122 & $20(16.4)$ & $2.2(0.9-5.1)$ & 0.080 & $8(6.6)$ & $2.8(0.6-13.4)$ & 0.186 \\
\hline 4 & 220 & $61(27.7)$ & $4.0(1.8-8.7)$ & 0.001 & $30(13.6)$ & $6.7(1.6-28.3)$ & 0.009 \\
\hline \multicolumn{8}{|l|}{ RDBN-K } \\
\hline 1 & 86 & $7(8.1)$ & 1.0 & & $2(2.3)$ & 1.0 & \\
\hline 2 & 97 & $13(13.4)$ & $1.7(0.7-4.4)$ & 0.239 & $3(3.1)$ & $1.3(0.2-8.1)$ & 0.744 \\
\hline
\end{tabular}

$\mathrm{P}<0.05$ is considered significant. RDBN, residual disease in breast and nodes; RDBN-K, residual disease in breast and nodes combined with $\mathrm{Ki}-67$; RR, relative risk; $\mathrm{Cl}$, confidence interval.

Table 6 Reclassification of RDBN categories using RDBN-K

\begin{tabular}{lccccc}
\hline \multirow{2}{*}{$\begin{array}{l}\text { RDBN } \\
\text { classification }\end{array}$} & No. & \multicolumn{4}{c}{ RDBN-K reclassification, No. (\%) } \\
\cline { 3 - 6 } & & 1 & 2 & 3 & 4 \\
\hline 1 & 86 & 86 & 0 & 0 & 0 \\
2 & 122 & 0 & 82 & 40 & 0 \\
3 & 295 & 0 & 15 & 278 & 2 \\
4 & 220 & 0 & 0 & 40 & 180 \\
\hline
\end{tabular}

RDBN, residual disease in breast and nodes; RDBN-K, residual disease in breast and nodes combined with $\mathrm{Ki}-67$. of 122 cases were reclassified to RDBN-K-3. Among cases that were originally RDBN-3, 17 (2.4\%) of 295 cases were reclassified, among which 15 cases were downgraded to RDBN-K-2 while 2 became RDBN-K-4. Among the 220 cases who were originally in RDBN-4, 40 (5.5\%) became RDBN-K-3. Over the entire follow-up period, $13.6 \%$ of patients in the RDBN-4 category deceased, and $15.9 \%$ of patients in the RDBN-K-4 category passed away (Figure 2).

Table 3 shows the prognostic analysis and comparison of 

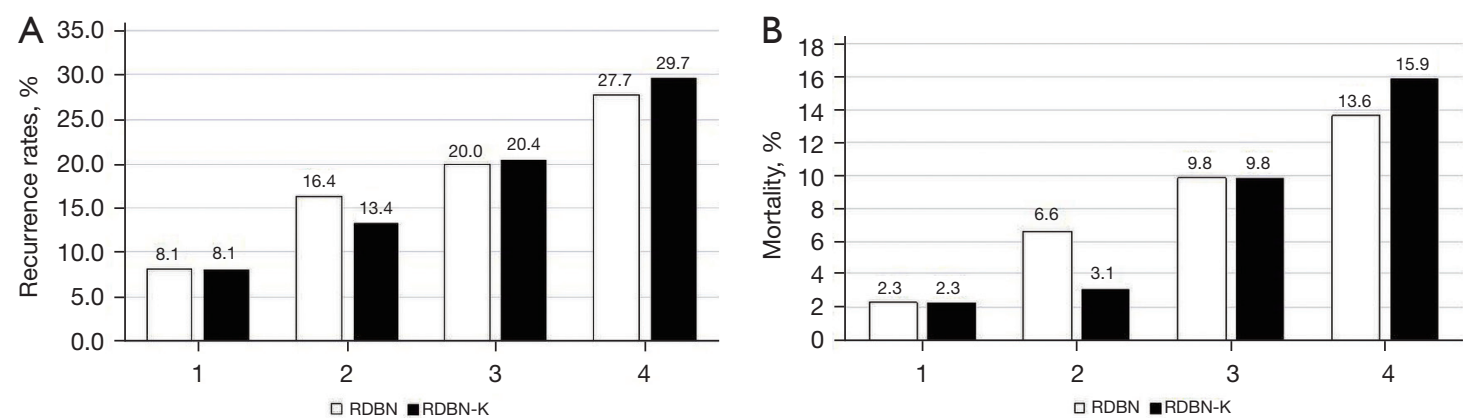

Figure 2 Recurrence rates (A) and mortality (B) by RDBN and RDBN-K. RDBN, residual disease in breast and nodes; RDBN-K, residual disease in breast and nodes combined with $\mathrm{Ki}-67$.

RDBN and RDBN-K. The 3-year DFS rates of patients with grades 1 to 4 in RDBN were $91.5 \%, 83.4 \%, 80.9 \%$ and $72.5 \%$ respectively, and the 3 -year OS rates were $98.8 \%, 96.6 \%, 93.9 \%$ and $90.0 \%$, respectively. The 3 -year DFS rates of patients with grades 1 to 4 in RDBN-K were $91.5 \%, 87.4 \%, 80.0 \%$, and $70.6 \%$, and the 3 -year OS rates were $98.8 \%, 99.0 \%, 94.1 \%$, and $87.9 \%$, respectively. Kaplan-Meier analysis for DFS and OS for all cases studied is depicted in Figure 3. RDBN was statistically significant for DFS and OS in all patients (DFS, $\mathrm{P}=0.001$; OS, $\mathrm{P}=0.006)$. RDBN-K was statistically significant for DFS and OS in all patients (DFS, $\mathrm{P}<0.001$; OS, $\mathrm{P}<0.001$ ). Tables 7,8 show the results of pairwise stratified analysis. No significant difference was found in the DFS and OS of patients between RDBN-1 and RDBN-2 and between RDBN-K-1 and RDBN-K-2. Although the survival curves of the DFS and OS of the two assessment methods were somewhat similar, the DFS and OS between patients in RDBN-K-3 and RDBN-K-4 were significantly significant (DFS, $\mathrm{P}=0.019$; OS, $\mathrm{P}=0.035$ ). On the other hand, the survival differences between RDBN-3 and RDBN-4 patients did not reach statistical significance (DFS, $\mathrm{P}=0.052$; OS, $\mathrm{P}=0.214)$. Similarly, the OS between RDBN-K-2 and RDBN-K-3 patients was statistically different $(\mathrm{P}=0.023)$ but that between RDBN-2 and RDBN-3 was not statistically significant $(\mathrm{P}=0.157)$.

\section{Discussion}

Systemic therapies (chemotherapy and hormonal therapy) of breast cancer are designed to reduce the risk of recurrence and improve overall survival. NAC for breast cancer can assess the response of the primary tumor to chemotherapy and provide opportunities to select the optimal therapeutic strategy when the tumor developing drug resistance shows stable disease (SD) and progressive disease (PD). Additionally, studies have shown that the pathological response of primary tumors to NAC is an important prognostic indicator $(1,2)$. Patients who achieved pCR were associated with a better prognosis than those with residual disease (9). Although the prognosis of patients with a lower RCB after NAC is not worse than that of $\mathrm{pCR}$ patients, the prognosis of patients with other degrees of residual disease remains unclear. Additionally, the clinical studies of both CREATE-X (20) and KATHERINE (21) confirmed that subsequent intensive treatment of patients who have not reached pCR after NAC could obtain a good prognosis. Therefore, an accurate assessment of the degree of pathological response and residual lesions on surgically resected specimens of patients after NAC can provide important prognostic information for clinicians to formulate subsequent treatment plans.

RCB was first described by Symmans et al. in 2007 (22). This method, recommended as the preferred method for use in clinical trials (23), uses the length and width $(\mathrm{mm})$ of the maximum two-dimensional cross section of the tumor bed, percentage of residual tumor cells in the tumor bed (\%), proportion of carcinoma in situ (\%), number of lymph node metastases and maximum diameter of the lymph node metastasis ( $\mathrm{mm}$ ) to calculate an RCB index. Patients are subsequently divided into four risk groups to assess the residual lesions after NAC to predict prognosis. These individual factors used by RDBN have been independently demonstrated to display prognostic value after NAC. These factors include the number of lymph nodes involved, residual breast tumors and tumor grade (6). Corben et al. (11) reported that age-unadjusted RDBN is still closely related to the prognosis of patients. Compared with $\mathrm{RCB}$, 

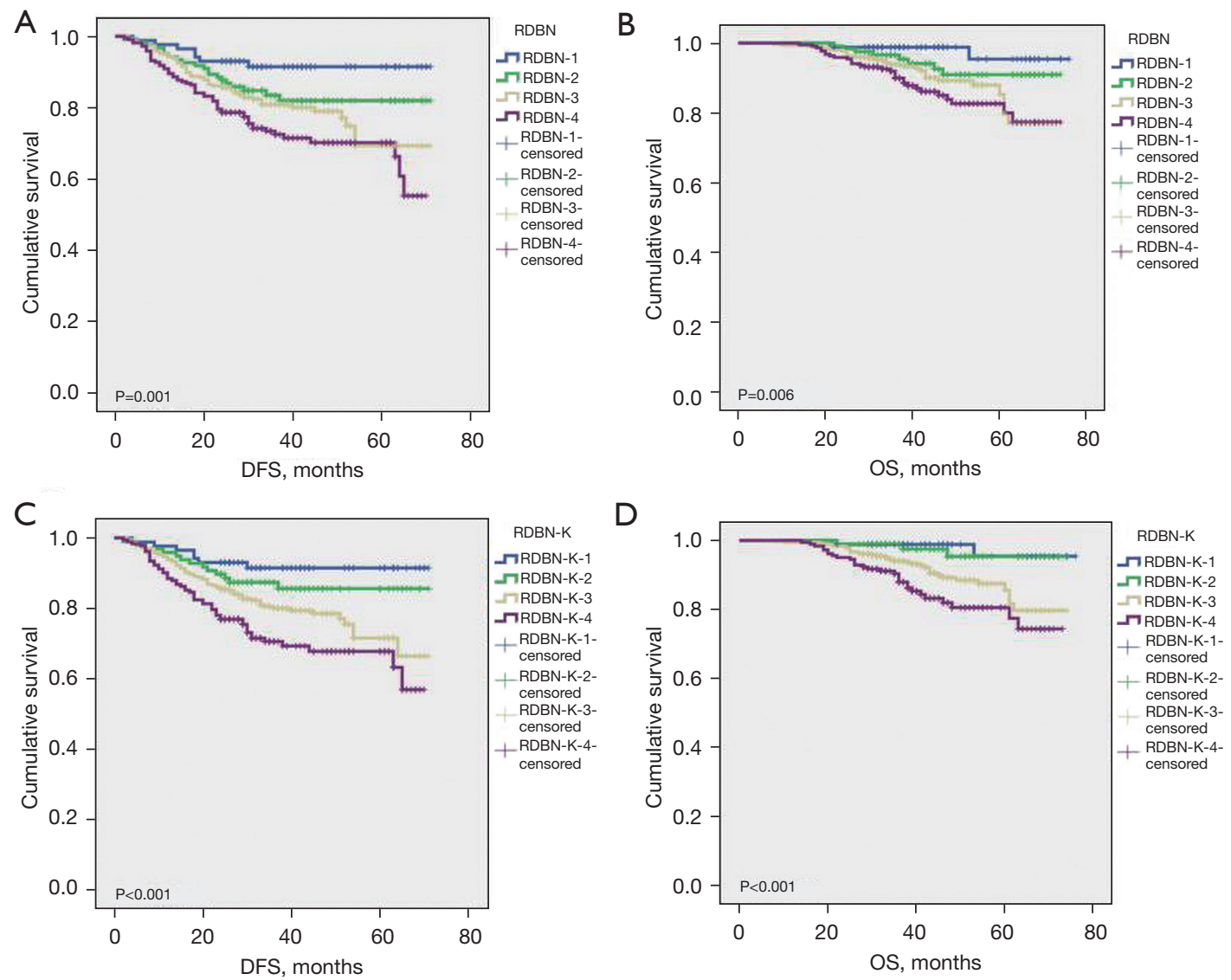

Figure 3 Kaplan-Meier survival curves of RDBN and RDBN-K. DFS (A,C) and OS (B,D) for all cases using RDBN (A,B) and RDBN-K (C,D) calculation. DFS, disease-free survival; OS, overall survival; RDBN, residual disease in breast and nodes; RDBN-K, residual disease in breast and nodes combined with Ki-67.

RDBN has added histological classification of tumors. RDBN emphasizes that the histological grade is the postchemotherapy histological grade because chemotherapy has an impact on histological grade and post-chemotherapy histological grade is an independent prognostic factor (24). The univariate prognostic analysis in this study showed that the histological grade of tumors is closely related to the prognosis of patients. Previous studies have also shown that patients with poorly differentiated tumors (grade II and grade III) after treatment have a worse prognosis than those with highly differentiated grade I tumors (24-26). This finding suggests that including tumor grade after treatment as a prognostic factor could help prognosis prediction of post-NAC patients with residual disease. A recent study (5) showed that although RCB has a better correlation in OS compared with RDBN, the accurate assessment of tumor bed size, cell properties, and proportion of carcinoma in situ requires more careful microscopic examination and broader sampling. Furthermore, cell enumeration is subjective and could be different between different observers (27). Histological grading is often evaluated in clinical practice. Therefore, concerning clinical feasibility, RDBN may be simpler and easier to use as an assessment method for residual lesions after NAC.

Currently, most studies focus on the correlation between the expression of $\mathrm{Ki}-67$ and pCR; only a few studies have analyzed the expression of $\mathrm{Ki}-67$ in residual lesions after NAC. In these studies, patients with a better response to NAC often showed a decrease in $\mathrm{Ki}-67$ expression (14). We speculate that the decrease in $\mathrm{Ki}-67$ expression is due to chemotherapy-sensitive cancer cells being mostly killed during the neoadjuvant period. The Ki-67 value reflects the percentage of proliferating cells in the tumor (28); therefore, $\mathrm{Ki}-67$ expression in tumors is lower in patients with a lower 
Table 7 Pairwise stratified analysis of RDBN with DFS and OS

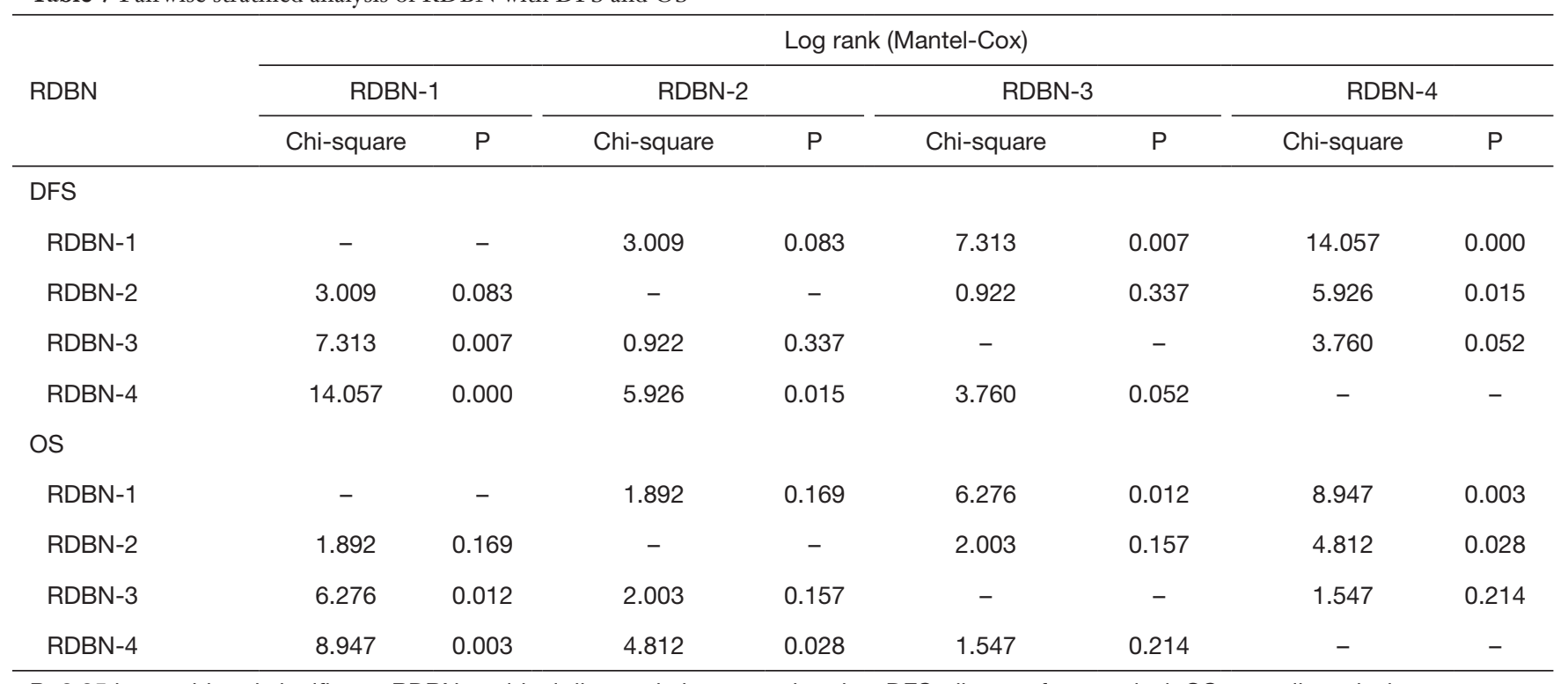

$\mathrm{P}<0.05$ is considered significant. RDBN, residual disease in breast and nodes; DFS, disease-free survival; OS, overall survival.

Table 8 Pairwise stratified analysis of RDBN-K with DFS and OS

\begin{tabular}{|c|c|c|c|c|c|c|c|c|}
\hline \multirow{3}{*}{ RDBN-K } & \multicolumn{8}{|c|}{ Log rank (Mantel-Cox) } \\
\hline & \multicolumn{2}{|c|}{ RDBN-K-1 } & \multicolumn{2}{|c|}{ RDBN-K-2 } & \multicolumn{2}{|c|}{ RDBN-K-3 } & \multicolumn{2}{|c|}{ RDBN-K-4 } \\
\hline & Chi-square & $\mathrm{P}$ & Chi-square & $\mathrm{P}$ & Chi-square & $P$ & Chi-square & $P$ \\
\hline \multicolumn{9}{|l|}{ DFS } \\
\hline RDBN-K-1 & - & - & 1.291 & 0.256 & 7.778 & 0.005 & 15.466 & 0.000 \\
\hline RDBN-K-3 & 7.778 & 0.005 & 2.713 & 0.100 & - & - & 5.539 & 0.019 \\
\hline RDBN-K-4 & 15.466 & 0.000 & 9.291 & 0.002 & 5.539 & 0.019 & - & - \\
\hline \multicolumn{9}{|l|}{ OS } \\
\hline RDBN-K-1 & - & - & 0.109 & 0.742 & 5.819 & .016 & 11.120 & 0.001 \\
\hline RDBN-K-4 & 11.120 & 0.001 & 10.992 & 0.001 & 4.423 & 0.035 & - & - \\
\hline
\end{tabular}

$\mathrm{P}<0.05$ is considered significant. RDBN-K, residual disease in breast and nodes combined with Ki-67; DFS, disease-free survival; OS, overall survival.

residual load. Several different cut-off points for Ki-67 have been proposed. The St. Gallen guide sets the critical value of Ki-67 expression at 14\% (29). The results of this study showed that Ki-67 expression after NAC is an independent prognostic factor for survival. Compared with patients with Ki-67 $\geq 14 \%$ after NAC, patients with Ki-67 $<14 \%$ showed better DFS and OS. Therefore, setting the critical value of $\mathrm{Ki}-67$ as $14 \%$ can provide accurate prognosis information. Other studies confirmed that patients with high Ki-67 values in residual tumors after chemotherapy had a worse prognosis $(12,15,17,30)$. Therefore, we combined the expression of Ki-67 after NAC with the residual tumor size, 
lymph node metastasis status and histological grade after NAC to form a new pathological assessment method called RDBN-K.

Overall, both RDBN and RDBN-K calculations provided prognostic information for DFS and OS in 723 cases of breast carcinoma receiving NAC treatment. NAC. The relative risk of recurrence and mortality increased with increasing classification, but RDBN-K has unique advantages. Pairwise stratified analysis (Tables 7,8) revealed that the OS difference between RDBN-K-2 and RDBN-K-3 was greater than that between RDBN-2 and RDBN-3. Similarly, differences in DFS and OS between grades 3 and 4 in RDBN-K stratification were greater than those between RDBN-3 and RDBN-4. In Table 7, comparing the evaluation results of the two pathological evaluation systems, we found that 97 (13\%) patients were reclassified. We believe that using the RDBN-K classification further selects patients with a mild residual load and better prognosis (RDBN-K-2) and severe residual load and poorer prognosis (RDBN-K-4). Therefore, the prognostic significance of grade 4 classifications calculated using RDBN-K may be more significant in predicting patient outcomes.

In conclusion, $\mathrm{Ki}-67$ expression after NAC is a standalone prognostic factor for breast cancer patients. The RDBN-K system can be used to understand the residual tumor burden and prognosis in breast cancer patients after NAC. Compared to RDBN, RDBN-K can provide a more accurate prognosis for breast cancer patients after neoadjuvant treatment. Therefore, based on the results of RDBN-K, follow-up therapy can be given to individual patients with a poor prognosis. For example, after neoadjuvant therapy based on anthracyclines, further treatment with taxanes can be administered to patients with a poor prognosis; additionally, it is possible to avoid adjuvant chemotherapy to improve the quality of life for patients with a good prognosis.

This study has the advantages of a large sample size, a standardized acquisition process of breast samples after NAC, and available detailed clinical and pathological data. However, it also has certain limitations. First, this study is retrospective, and presently, no standardized Ki67 assessment system exists. The consistency of the Ki67 index assessment by different pathologists may be poor. Second, neither RDBN nor RDBN-K is suitable for patients who have not undergone axillary lymph node dissection because one of its most important components is the metastatic status of lymph nodes after NAC. It is critical for the surgeon to obtain the pretherapy positive lymph node status at the time of resection and for the pathologist to record the existence of a biopsy clip and/or biopsy site to ensure that the excised lymph node is actually the previous positive lymph node. Therefore, prospective studies and long-term follow-up in large-cohort populations are needed to obtain evidence to support our results.

\section{Acknowledgments}

Funding: None.

\section{Footnote}

Reporting Checklist: The authors have completed the REMARK reporting checklist (available at https://tbcr. amegroups.com/article/view/10.21037/tbcr-21-39/rc).

Data Sharing Statement: Available at https://tbcr.amegroups. com/article/view/10.21037/tbcr-21-39/dss

Conflicts of Interest: All authors have completed the ICMJE uniform disclosure form (available at https:// tbcr.amegroups.com/article/view/10.21037/tbcr-21-39/ coif). CG serves as an unpaid editorial board member of Translational Breast Cancer Research from March 2020 to February 2022, and YL serves as an unpaid editorial board member of Translational Breast Cancer Research from May 2021 to April 2023. The other authors have no conflicts of interest to declare.

Ethical Statement: The authors are accountable for all aspects of the work in ensuring that questions related to the accuracy or integrity of any part of the work are appropriately investigated and resolved. The study was conducted in accordance with the Declaration of Helsinki (as revised in 2013). This study was approved by Institutional Review Board of The Fourth Hospital of Hebei Medical University (No. 2020KY112). Informed consent was obtained from all individual participants included in the study.

Open Access Statement: This is an Open Access article distributed in accordance with the Creative Commons Attribution-NonCommercial-NoDerivs 4.0 International License (CC BY-NC-ND 4.0), which permits the noncommercial replication and distribution of the article with the strict proviso that no changes or edits are made and the 
original work is properly cited (including links to both the formal publication through the relevant DOI and the license). See: https://creativecommons.org/licenses/by-nc-nd/4.0/.

\section{References}

1. Bonadonna G, Valagussa P, Brambilla C, et al. Primary chemotherapy in operable breast cancer: eight-year experience at the Milan Cancer Institute. J Clin Oncol 1998;16:93-100.

2. Kuerer HM, Newman LA, Smith TL, et al. Clinical course of breast cancer patients with complete pathologic primary tumor and axillary lymph node response to doxorubicin-based neoadjuvant chemotherapy. J Clin Oncol 1999;17:460-9.

3. Bear HD, Anderson S, Smith RE, et al. Sequential preoperative or postoperative docetaxel added to preoperative doxorubicin plus cyclophosphamide for operable breast cancer:National Surgical Adjuvant Breast and Bowel Project Protocol B-27. J Clin Oncol 2006;24:2019-27.

4. Hennessy BT, Hortobagyi GN, Rouzier R, et al. Outcome after pathologic complete eradication of cytologically proven breast cancer axillary node metastases following primary chemotherapy. J Clin Oncol 2005;23:9304-11.

5. Choi M, Park YH, Ahn JS, et al. Assessment of pathologic response and long-term outcome in locally advanced breast cancers after neoadjuvant chemotherapy: comparison of pathologic classification systems. Breast Cancer Res Treat 2016;160:475-89.

6. Chollet P, Abrial C, Durando X, et al. A new prognostic classification after primary chemotherapy for breast cancer: residual disease in breast and nodes (RDBN). Cancer J 2008;14:128-32.

7. Zhao Y, Dong X, Li R, et al. Evaluation of the pathological response and prognosis following neoadjuvant chemotherapy in molecular subtypes of breast cancer. Onco Targets Ther 2015;8:1511-21.

8. Mombelli S, Kwiatkowski F, Abrial C, et al. Prognostic factors in operable breast cancer treated with neoadjuvant chemotherapy: towards a quantification of residual disease. Oncology 2015;88:261-72.

9. Clark BZ, Farrugia DJ, Landmann A, et al. Prognostic Significance of Modified Residual Disease in Breast and Nodes (mRDBN) Algorithm After Neoadjuvant Chemotherapy for Breast Cancer. Am J Clin Pathol 2018;149:332-43.

10. Lee HJ, Park IA, Song IH, et al. Comparison of Pathologic
Response Evaluation Systems after Anthracycline with/ without Taxane-Based Neoadjuvant Chemotherapy among Different Subtypes of Breast Cancers. PLoS One 2015;10:e137885.

11. Corben AD, Abi-Raad R, Popa I, et al. Pathologic response and long-term follow-up in breast cancer patients treated with neoadjuvant chemotherapy: a comparison between classifications and their practical application. Arch Pathol Lab Med 2013;137:1074-82.

12. Ingolf JB, Russalina M, Simona M, et al. Can ki67 play a role in prediction of breast cancer patients' response to neoadjuvant chemotherapy? Biomed Res Int 2014;2014:628217.

13. Miglietta L, Morabito F, Provinciali N, et al. A prognostic model based on combining estrogen receptor expression and $\mathrm{Ki}-67$ value after neoadjuvant chemotherapy predicts clinical outcome in locally advanced breast cancer: extension and analysis of a previously reported cohort of patients. Eur J Surg Oncol 2013;39:1046-52.

14. von Minckwitz G, Schmitt WD, Loibl S, et al. Ki67 measured after neoadjuvant chemotherapy for primary breast cancer. Clin Cancer Res 2013;19:4521-31.

15. Yoshioka T, Hosoda M, Yamamoto M, et al. Prognostic significance of pathologic complete response and Ki67 expression after neoadjuvant chemotherapy in breast cancer. Breast Cancer 2015;22:185-91.

16. Jones RL, Salter J, A'Hern R, et al. The prognostic significance of Ki67 before and after neoadjuvant chemotherapy in breast cancer. Breast Cancer Res Treat 2009;116:53-68.

17. Montagna E, Bagnardi V, Viale G, et al. Changes in $\operatorname{PgR}$ and $\mathrm{Ki}-67$ in residual tumour and outcome of breast cancer patients treated with neoadjuvant chemotherapy. Ann Oncol 2015;26:307-13.

18. Elston CW, Ellis IO. Pathological prognostic factors in breast cancer. I. The value of histological grade in breast cancer: experience from a large study with long-term follow-up. Histopathology 1991;19:403-10.

19. Dowsett M, Nielsen TO, A'Hern R, et al. Assessment of Ki67 in breast cancer: recommendations from the International Ki67 in Breast Cancer working group. J Natl Cancer Inst 2011;103:1656-64.

20. Zujewski JA, Rubinstein L. CREATE-X a role for capecitabine in early-stage breast cancer: an analysis of available data. NPJ Breast Cancer 2017;3:27.

21. von Minckwitz G, Huang CS, Mano MS, et al. Trastuzumab Emtansine for Residual Invasive HER2Positive Breast Cancer. N Engl J Med 2019;380:617-28. 
22. Symmans WF, Peintinger F, Hatzis C, et al. Measurement of residual breast cancer burden to predict survival after neoadjuvant chemotherapy. J Clin Oncol 2007;25:4414-22.

23. Provenzano E, Bossuyt V, Viale G, et al. Standardization of pathologic evaluation and reporting of postneoadjuvant specimens in clinical trials of breast cancer: recommendations from an international working group. Mod Pathol 2015;28:1185-201.

24. Amat S, Penault-Llorca F, Cure H, et al. Scarff-BloomRichardson (SBR) grading: a pleiotropic marker of chemosensitivity in invasive ductal breast carcinomas treated by neoadjuvant chemotherapy. Int J Oncol 2002;20:791-6.

25. Simpson JF, Gray R, Dressler LG, et al. Prognostic value of histologic grade and proliferative activity in axillary node-positive breast cancer: results from the Eastern Cooperative Oncology Group Companion Study, EST 4189. J Clin Oncol 2000;18:2059-69.

26. Latinovic L, Heinze G, Birner P, et al. Prognostic relevance of three histological grading methods in breast

doi: $10.21037 /$ tbcr-21-39

Cite this article as: Han R, Ma Y, Liu Z, Li C, Li S, Kong X, Han M, Liu Y, Geng C. A new pathological assessment method to assess residual lesions after neoadjuvant chemotherapy for breast cancer, residual disease in breast and nodes combined with Ki-67 (RDBN-K). Transl Breast Cancer Res 2022;3:24. cancer. Int J Oncol 2001;19:1271-7.

27. Rajan R, Poniecka A, Smith TL, et al. Change in tumor cellularity of breast carcinoma after neoadjuvant chemotherapy as a variable in the pathologic assessment of response. Cancer 2004;100:1365-73.

28. Rossi L, Laas E, Mallon P, et al. Prognostic impact of discrepant Ki67 and mitotic index on hormone receptorpositive, HER2-negative breast carcinoma. Br J Cancer 2015;113:996-1002.

29. Goldhirsch A, Wood WC, Coates AS, et al. Strategies for subtypes--dealing with the diversity of breast cancer: highlights of the St. Gallen International Expert Consensus on the Primary Therapy of Early Breast Cancer 2011. Ann Oncol 2011;22:1736-47.

30. Yamazaki N, Wada N, Yamauchi C, et al. High expression of post-treatment $\mathrm{Ki}-67$ status is a risk factor for locoregional recurrence following breast-conserving surgery after neoadjuvant chemotherapy. Eur J Surg Oncol 2015;41:617-24. 